\title{
Molecular Dynamics Simulations of the Role of Salinity and Temperature on the Hydrocarbon/Water Interfacial Tension
}

Elena R. Remesal, Javier Amaya Suárez, Antonio M. Márquez and Javier Fdez. Sanz

Dep. of Physical Chemistry, University of Seville, 41012 Seville, (Spain)

Cristina Rincón and José Guitián

Technology Repsol S.A. Madrid, (Spain)

\begin{abstract}
Interfacial tension of some hydrocarbon/water systems, including a mixture of aliphatic and aromatic hydrocarbons, has been estimated on the basis of molecular dynamics simulations. The dependence of the interfacial properties on the salinity of the aqueous phase and the temperature has been simulated. Different concentrations in $\mathrm{NaCl}$ and $\mathrm{CaCl}_{2}$ up to $2 \mathrm{M}$ have been used. It is found that, in all considered cases, interfacial tension increases with salt concentration. This effect depends on the preference of the salt ions for the bulk of the aqueous phase that, in turns, results in an increased difficulty for the water molecules to be at the interface. The influence of salinity is fundamentally electrostatic in origin and does not depends on the chemical nature of the salt cation. Finally, the impact of temperature on the dodecane/brine interfacial tension has also been inspected. A decreasing of the values of the interfacial tension is found in agreement, both in trend and magnitude, with experimental available data.
\end{abstract}




\section{Introduction}

Prediction of interfacial properties, especially interfacial tension (IFT) between immiscible and partially miscible liquid-liquid mixtures, is of great interest among the scientific community. Thermodynamically, the IFT is a measure of the free energy per unit area that is required for creating an interface between two condensed phases. It plays a key role in a number of applications such as emulsion stability, coating, micelle formation and self-assembly process of nanoparticles at liquid-liquid interfaces. It is also relevant in pharmaceutical and cosmetic industry. In petroleum industry, interaction between oil components, water and rock is a key role in enhanced-oil-recovery process (EOR).

Experimentally, IFT can be measured by several techniques, as capillary, Wilhelmy plates, drop weight, gas bubble, spinning drop, pendant drop and ring methods. Alternatively, theoretical methods based on atomistic molecular dynamics (MD) simulations have been used for long. MD simulations also using coarse-grained approaches or dissipative particle dynamics (DPD) have been reported. [1, 2, 3]

Different trends have been reported in the literature regarding the effects of the presence of diverse salts on the interfacial tension of water/oil systems. In general, in pure hydrocarbon/water systems, an increase of the IFT with salt concentration has been described [4,5] with the notable exception of KI, for which a decreasing trend was found for dodecane/water systems [6]. The interfacial tension of crude oil/brine systems seems to be, however, a complex function of brine composition and concentration. Some studies $[7,8]$ have reported an initial decrease with later linear increase of the IFT with brine concentration, resulting in the existence of a critical brine concentration that minimizes the IFT. However, other studies have reported no significant influence of $\mathrm{NaCl}$ concentration on the IFT [9] and even a decrease of the IFT with increasing salinity of the aqueous phase [10].

Computer simulation techniques have been extensively used in recent years as useful complements of experimental studies of the properties of oil/brine interfaces. Previous MD works have usually considered pure organic solvents as the oil phase $[11,12,13,14]$. However, more recently, complex mixtures consisting of alkanes, cycloalkanes and aromatic molecules [15,16] even including an oil polar fraction [17] have been considered, recognizing the importance that the composition has in the 
properties of crude oils. These theoretical simulations allow us to understand structure and properties of the interface.

In this research, interfacial tensions (IFT) are estimated for two immiscible liquid phases: a water solution and an oil phase, through molecular dynamics simulations and dissipative particle dynamics. As oil we have employed dodecane and octane (as aliphatic hydrocarbons) and benzene and toluene (as aromatic hydrocarbons). The dependence of the interfacial properties on both physical and chemical variables constitutes one of the most appealing aspects of these interfaces. To examine this dependence, the effects of different water salinities and temperature have also been analyzed.

\section{Computational details.}

In this work different types of simulations such as atomistic molecular dynamics (MD) and dissipative particle dynamics (DPD) have been used to compute the IFT of different aqueous solution-hydrocarbon systems. All calculations were performed at a pressure of $1 \mathrm{~atm}$ and $\mathrm{T}=295.15 \mathrm{~K}$, unless otherwise indicated, using three dimensional periodic boundary conditions. The pressure tensor method was used to compute the interfacial tension. With the interface located perpendicular to the $z$-axis, as in our case, the interfacial tension, $\gamma$, can be obtained from the diagonal components of the pressure tensor, $P_{i i}$, as: [18]

$$
\gamma=\frac{L_{z}}{2}\left(P_{z z}-\frac{P_{x x}+P_{y y}}{2}\right)
$$

where $L_{z}$ is the length of the simulation box along the $z$-axis, and the $1 / 2$ factor is included to account for the two interfaces in the simulation box.

Atomistic molecular dynamics (MD) simulations were performed with the Large Atomic/Molecular Massively Parallel Simulator (LAMMPS) package [19]. The consistent valence force field (CVFF) [20], which is known to correctly describe the interactions between different organic molecules, was used for all the systems. A cutoff distance of $1.4 \mathrm{~nm}$ for the Lennard-Jones potential was employed together with a van der Waals tail correction. It was checked that lower cutoff distances resulted in wrong densities for the isolated organic or water phases (vide infra) and, as result, lower values 
for the IFT were obtained. In example, a cutoff distance of $0.8 \mathrm{~nm}$ produces IFT values $10 \%$ lower than those obtained with the much tight cutoff of $1.4 \mathrm{~nm}$ employed. The long range electrostatic interactions were handled with the particle-particle-particlemesh (PPPM) method with an accuracy of 1 part in $10^{5}$ [21]. To integrate the Newton's equations of motion a time step of $1.0 \mathrm{fs}$ was used unless stated otherwise. All the production simulations were carried out in the NVT ensemble using a Nosé-Hoover thermostat set at a temperature of $\mathrm{T}=295.15 \mathrm{~K}$ [22].

The simulation box is an orthorhombic cell of dimensions $7 \times 7 \times 10 \mathrm{~nm}^{3}$ for systems where the organic phase is a pure component and $8.5 \times 8.5 \times 16.0 \mathrm{~nm}^{3}$ for systems where the organic phase is $87 / 13 \%$ wt dodecane/toluene (DodTol) (Fig. 1a). The organic phase is always situated at the center and the remaining volume is filled with the aqueous phase, either pure water or the saline solution $\left(\mathrm{NaCl}\right.$ or $\left.\mathrm{CaCl}_{2}\right)$. In the case of the DodTol/ $\mathrm{NaCl} 2.0 \mathrm{M}$ brine, for example, this results in 1375 dodecane and 375 toluene molecules on the oil phase, and 19444 water molecules, $700 \mathrm{Na}^{+}$and $700 \mathrm{Cl}^{-}$ ions on the aqueous phase. The models were built using the Packmol code [23]. In order to validate the simulation box size, force field and general setup simulation parameters a preliminary set of simulations were performed to obtain physical properties of water and isolated hydrocarbons. In these preliminary calculations, the systems were first equilibrated in a 2 ns NPT simulation and, later, a second production run of 4 ns was used to average the system properties. This way, we were able to compare the computed isolated phase density with reported experimental data and adjust the simulation setup. A similar procedure was used to compute the IFT of the two phase systems. First, the two phase system was equilibrated on a 2 ns NPT run. Later, in a 10 ns NVT production run, the system properties were obtained as average magnitudes each 1 ns by sampling the trajectories every $1 \mathrm{fs}$. After checking that the IFT has reached a stable value, the final IFT value is obtained as the average of the last 3 ns and the standard deviation of this average $\left( \pm 0.2 \mathrm{mN} \mathrm{m}^{-1}\right)$ gave us an estimation of the precision in the calculated IFT.

DPD simulations were carried out with Material Studio 8.0 [24] using the Mesocite-DPD module [25, 26]. The box size and the number of beads taken to represent the organic and water phases are reported in Table 1. Cell compositions were fixed to be mixture 50:50 binary components. Several properties such as density, isothermal compressibility, and solubility parameters of an individual component are 
taken from Martini et al. [27] and Rezaei et al. [28]. An equilibration period of 50000 steps was used and followed by a production run of 50000 steps.

\section{Results and discussion}

Calculated interfacial tension values for the organic phase/water systems are summarized in Table 2 and Fig. 2, both at the MD (Table 2 and Fig. 2a) and DPD (Table 2 and Fig. 2b) approaches. Molecular dynamics results show a sharp interface in all cases, as can be deduced from the representation of the water and organic phase molecular density along the direction perpendicular to the interface (see Fig. 3). This observation is consistent with the experimental insolubility of these organic phases and water. Calculated interfacial tension for the aliphatic phase/water systems is significantly higher than for the aromatic phases/water systems reflecting, again, the low water solubility of these compounds and that some favorable interaction builds up in the case of the aromatics/water interface. The MD calculated interfacial tensions are systematically lower than the experimental values reported in the literature by about $4 \%$ for the aliphatic/water systems. However, for the aromatics/water systems the computed IFTs are, now, slightly larger (by about 5\%) than the available experimental data that have, themselves, a dispersion of $2-3 \%$ on the reported figures. These errors are similar to those of other theoretical MD calculations reported in the literature and have to be traced to the force field parameters, as we have checked that our quite tight MD setup consistently results in a dispersion of the average IFT calculated values of only $\pm 0.2 \mathrm{mN} \cdot \mathrm{m}^{-1}$ (vide supra). The DPD results show a similar behavior: the organic phases/water interfaces are quite sharp in agreement with the low miscibility of these systems. The computed interfacial tension data are, now, in better agreement with the experimental values and show a much better agreement that previously reported DPD results $[27,28,29]$

Regarding our DodTol/water system, the computed IFT is $49.9 \mathrm{mN} \cdot \mathrm{m}^{-1}$ (MD) and $46.0 \mathrm{mN} \cdot \mathrm{m}^{-1}$ (DPD). In this case, we have not found any previous experimental data, but given that the interfacial tension is directly related to the free energy required to the interface creation and considering the dodecane/toluene mix an ideal system, we estimated the value for the DodTol /water system from experimental values for the Dod/water and Tol/water systems as 


$$
\gamma_{D o d T o l / w}=x_{D} \gamma_{D / w}+x_{T} \gamma_{T / w}
$$

where $\gamma_{\mathrm{D} / \mathrm{W}}$ and $\gamma_{\mathrm{T} / \mathrm{W}}$ are the interfacial tension of pure dodecane/water and pure toluene/water interfaces, and $\mathrm{x}_{\mathrm{D}}$ and $\mathrm{x}_{\mathrm{T}}$ are the dodecane and toluene molar fractions, resulting in a value of $48.5 \mathrm{mN} \cdot \mathrm{m}^{-1}$ for this interface. Our calculated MD and DPD values show deviations from this value that are within the errors bars previously analyzed. However, we found noteworthy that the error for the MD calculated value has decreased while for the computed DPD value has increased. For this reason, we analyzed in detail the structure of the dodecane-toluene/water interface by plotting the molecular density for the different components along the direction perpendicular to the interface (Fig. 3). This plot shows, as expected, a sharp interface, but it also shows an increase of the toluene molecular density at the interface region with respect to the bulk organic phase, with the molecular toluene density at the interface nearly duplicating the bulk value. This means that the dodecane-toluene system does not behaves totally as an ideal system, and explains the behavior observed in the computed MD and DPD interfacial tension values. As toluene accumulates at the interface, the DodTol/water IFT is lower than what the ideal mixture formula (2) could predict, explaining the apparent behavior of the errors of the calculated IFT values. This accumulation of toluene at the organic phase/water interface has been already observed in other aromatics+aliphatics/water systems and has been traced to the presence of weak hydrogen bonds between the water molecules and the aromatics [15].

The computed IFTs between the oil phase (both pure organic solvents and dodecane-toluene mixture) and an aqueous solution as a function of salt composition and concentration are summarized in Table 3 and Fig. 4. The brine concentration was changed between $0.0-2.0 \mathrm{M}$ for both $\mathrm{NaCl}$ and $\mathrm{CaCl}_{2}$ and we examined the oil/water IFT for dodecane, toluene and the dodecane-toluene mixture. In all systems studied, the IFT increased with salt concentration in contrast with the recent results of Moeini et al. [8] that found a moderate decrease of the IFT of heavy crude oil and brine up to $~ 40000$ ppm for both $\mathrm{NaCl}$ and $\mathrm{CaCl}_{2}$ brine then, the IFT increased linearly with further addition of salt. This can be ascribed to the presence in their systems of a polar component, asphaltene, in the organic phase that accumulates at the interface and interacts favorably with the ions at low salt concentration. In our case, the aromatic component (toluene) accumulates at the interface (see Fig. 3) and influences the 
behavior of the IFT in the DodTol/brine systems. From the evolution of the IFT with $\mathrm{NaCl}$ concentration represented in Fig. 4 for isolated components, we can observe that the increase of IFT with molar concentration is large for dodecane/brine than for toluene/brine systems. For the DodTol/brine system, the slope of the IFT vs $\mathrm{NaCl}$ concentration curve is quite close to that of pure toluene/brine. This is a consequence of the above discussed accumulation of toluene at the interface in DodTol/water systems that is related to the formation of weak hydrogen bonds between the water molecules and the $\pi$-systems of the toluene molecules. The much higher concentration of the aromatic component at the interface results in a strong relationship of the DodTol/brine interface properties to those of the isolated toluene/brine systems. The slope of the IFT vs $\mathrm{CaCl}_{2}$ concentration is notably higher for both systems tested in this work: pure dodecane and dodecane-toluene/brine. The only experimental work that, to the best of our knowledge, has examined the individual effects of monovalent and divalent salt ions on oil/brine interfacial tension is the already mentioned study of Moeini et al. They found similar trends for both $\mathrm{NaCl}$ and $\mathrm{CaCl}_{2}$ solutions: IFT decreasing at low salt concentrations and then increasing at higher concentrations, but higher IFT values at all concentration were found when using $\mathrm{CaCl}_{2}$.

Our MD simulations allow us to further delve into the interface structure and analyze this behavior in terms of the lack of cation affinity for the interface. The normalized molecular density profiles along the perpendicular direction to the interface for the dodecane-toluene/ $\mathrm{NaCl} 2.0 \mathrm{M}$ system, shown in Fig. 3, show that the $\mathrm{Na}^{+}$cations are, effectively, not present at the interface but prefer to be fully solvated by water molecules at the bulk of the aqueous solution. This results, in parallel, in a less favorable situation for water molecules at the interface, where they weakly interact with toluene molecules. At the same time, in the bulk of the aqueous solution they interact much strongly with the salt cations. This interpretation allows us to understand the higher effect of the $\mathrm{Ca}^{2+}$ cations as they interact much strongly with the water molecules and they also have a larger coordination sphere.

We can, thus, assign to purely electrostatic interactions the apparent differential effect of the $\mathrm{CaCl}_{2}$ compared to the $\mathrm{NaCl}$. If the computed IFT is represented versus the ionic strength, as shown in Fig. $4 \mathrm{~b}$, data from both $\mathrm{NaCl}$ and $\mathrm{CaCl}_{2}$ aqueous solutions and dodecane or dodecane-toluene mixture fit in the same straight line. The slope of the fitting is, nonetheless, lower for the mixture than for the pure dodecane organic phase. 
This indicates that there is an effect related to the accumulation of toluene at the interface that has to be analyzed in detail in a future work. In fact, the effect of brine concentration that we observe is much lower than the data shown by Moieini et al. [8], something that is probably related to the presence of polar components in their organic phase that are polarized by the reorganization of the interface induced by the cations of the aqueous phase.

The effect of temperature has been investigated on the dodecane/brine interface at $323.15 \mathrm{~K}$ and concentrations of $0.0,0.2,1.0$, and $2.0 \mathrm{M}$ of $\mathrm{NaCl}$ (Fig. 5). It is found that increasing the temperature decreases the interfacial tension by $1-3 \mathrm{mN} \cdot \mathrm{m}^{-1}$. This is in agreement, both in trend and magnitude, with previous experimental work [31, 29, 8] as several authors have reported this same behavior. This is relevant, as the measurement of the IFT at high temperature is quite cumbersome and also because the high temperatures present of the crude reservoirs strongly influence the interaction between oil components, water and rock. Thus, we show that molecular dynamics techniques offer a competitive means of obtaining an understanding of the interfacial tension behavior in hydrocarbon/brine systems at high temperatures.

\section{Conclusions}

In this work we conducted a series of theoretical simulations based on molecular dynamics and dissipative particle dynamics to investigate the effect of salinity and temperature on the interfacial tension of various hydrocarbon/aqueous phase systems. We considered both pure hydrocarbons and a mixture of aliphatic and aromatic components and $\mathrm{NaCl}$ and $\mathrm{CaCl}_{2}$ as saline components to investigate the effect of cation valence on IFT. The interfacial tension of the different hydrocarbon/brine systems is found to rise with increasing salt concentration. This behavior is analyzed in terms of the electrolyte preference for the bulk aqueous phase, which, of course is related with its strong interaction with water molecules. The $\mathrm{Ca}^{2+}$ cation, that has a larger charge and coordination sphere, is found to have a superior effect on the IFT. However, the influence of the brine concentration, for the systems studied and at the salt concentrations examined, can be traced to a purely electrostatic influence, as the IFT increases linearly with the ionic strength on these systems. Rising the system temperature is found to decrease the computed dodecane/brine IFT, a trend that is in 
agreement with experimental findings, showing that computational techniques are a competitive way to estimate the IFT at the high temperature conditions present on oil reservoirs.

\section{Acknowledgments}

This work was funded by Repsol S.A., grant number 2264/0638. 
Table 1 Coarse graining in the DPD simulations.

\begin{tabular}{|l|c|c|c|c|}
\hline Oil phase & $\begin{array}{c}\text { Beads } \\
\text { hydrocarbon } \\
\text { molecule }\end{array}$ & $\begin{array}{c}\text { Water } \\
\text { molecules/bead }\end{array}$ & Cell size* & Total beads \\
\hline Octane & 3 & 3 & $19 \times 12 \times 12$ & 2880 \\
\hline Dodecane & 3 & 4 & $19 \times 12 \times 12$ & 2880 \\
\hline Benzene & 1 & 5 & $19 \times 9 \times 9$ & 1620 \\
\hline Toluene & 1 & 6 & $19 \times 12 \times 12$ & 2880 \\
\hline
\end{tabular}

*Reduced units

Table 2 IFT $\left(\mathrm{mN} \cdot \mathrm{m}^{-1}\right) \mathrm{MD}$ and DPD results for the hydrocarbon/water systems and literature data.

\begin{tabular}{|c|c|c|c|c|c|}
\hline \multirow{2}{*}{ Oil phase } & \multicolumn{2}{|c|}{ MD } & \multicolumn{2}{c|}{ DPD } & \multirow{2}{*}{ Experimental } \\
\cline { 2 - 6 } & This work & Literature & This work & Literature & \\
\hline Octane & 48.4 & $52.72^{\mathrm{a}} ; 53.3^{\mathrm{b}}$ & 52.8 & $\begin{array}{c}45.37^{\mathrm{c}} ; 50.12^{\mathrm{c}} ; \\
51.8^{\mathrm{d}} ; 49.2^{\mathrm{e}}\end{array}$ & $\begin{array}{c}51.45^{\mathrm{f}} ; 52.4^{\mathrm{g}} ; \\
50.8^{\mathrm{h}}\end{array}$ \\
\hline Dodecane & 50.5 & & 53.5 & $45.15^{\mathrm{c}} ; 56.6^{\mathrm{d}}$ & $\begin{array}{c}52.74^{\mathrm{f}} ; 53.7^{\mathrm{g}} ; \\
52.8^{\mathrm{h}}\end{array}$ \\
\hline Benzene & 36.8 & $34.42^{\mathrm{a}} ; 39.2^{\mathrm{b}} ; 38.5^{\mathrm{b}}$ & 35.4 & $\begin{array}{c}34.7^{\mathrm{c}} ; 31.42^{\mathrm{c}} ; \\
34.2^{\mathrm{d}} ; 34.7^{\mathrm{e}}\end{array}$ & $33.8^{\mathrm{h}} ; 35.0^{\mathrm{i}}$ \\
\hline Toluene & 38.3 & $37.69^{\mathrm{a}} ; 41.2^{\mathrm{b}} ; 40.4^{\mathrm{b}}$ & 37.1 & $32.65^{\mathrm{c}} ; 36.22^{\mathrm{c}}$ & $35.4^{\mathrm{h}} ; 36.1^{\mathrm{i}}$ \\
\hline DodTol & 49.9 & & 46.0 & & $49.0^{\mathrm{j}}$ \\
\hline
\end{tabular}

a)[15] b)[30]c) [28] d) [29] e) [27] f)[31] g) [32] h) [33] i) [34] j) [35]

Table 3 IFT values $\left(\mathrm{mN} \cdot \mathrm{m}^{-1}\right)$ for hydrocarbon/water vs brine concentration estimated from MD simulations.

\begin{tabular}{|c|c|c|c|c|}
\hline \multirow{2}{*}{ Oil phase } & \multicolumn{4}{|c|}{ C / M } \\
\cline { 2 - 5 } & $\mathbf{0 . 0}$ & $\mathbf{0 . 2}$ & $\mathbf{1 . 0}$ & $\mathbf{2 . 0}$ \\
\hline & \multicolumn{5}{|c|}{ NaCl } \\
\hline Dodecane & 50.5 & 50.7 & 54.3 & 56.0 \\
\hline Dodecane (T=323.15K) & 48.4 & 49.5 & 52.5 & 53.1 \\
\hline Toluene & 38.8 & 39.5 & 40.8 & 42.3 \\
\hline DodTol & 49.9 & 50.3 & 50.8 & 53.0 \\
\hline & & \multicolumn{3}{|c|}{ CaCl $_{\mathbf{2}}$} \\
\hline Dodecane & 50.5 & 53.1 & 56.9 & 60.3 \\
\hline DodTol & 49.9 & 50.9 & 55.4 & 57.2 \\
\hline
\end{tabular}



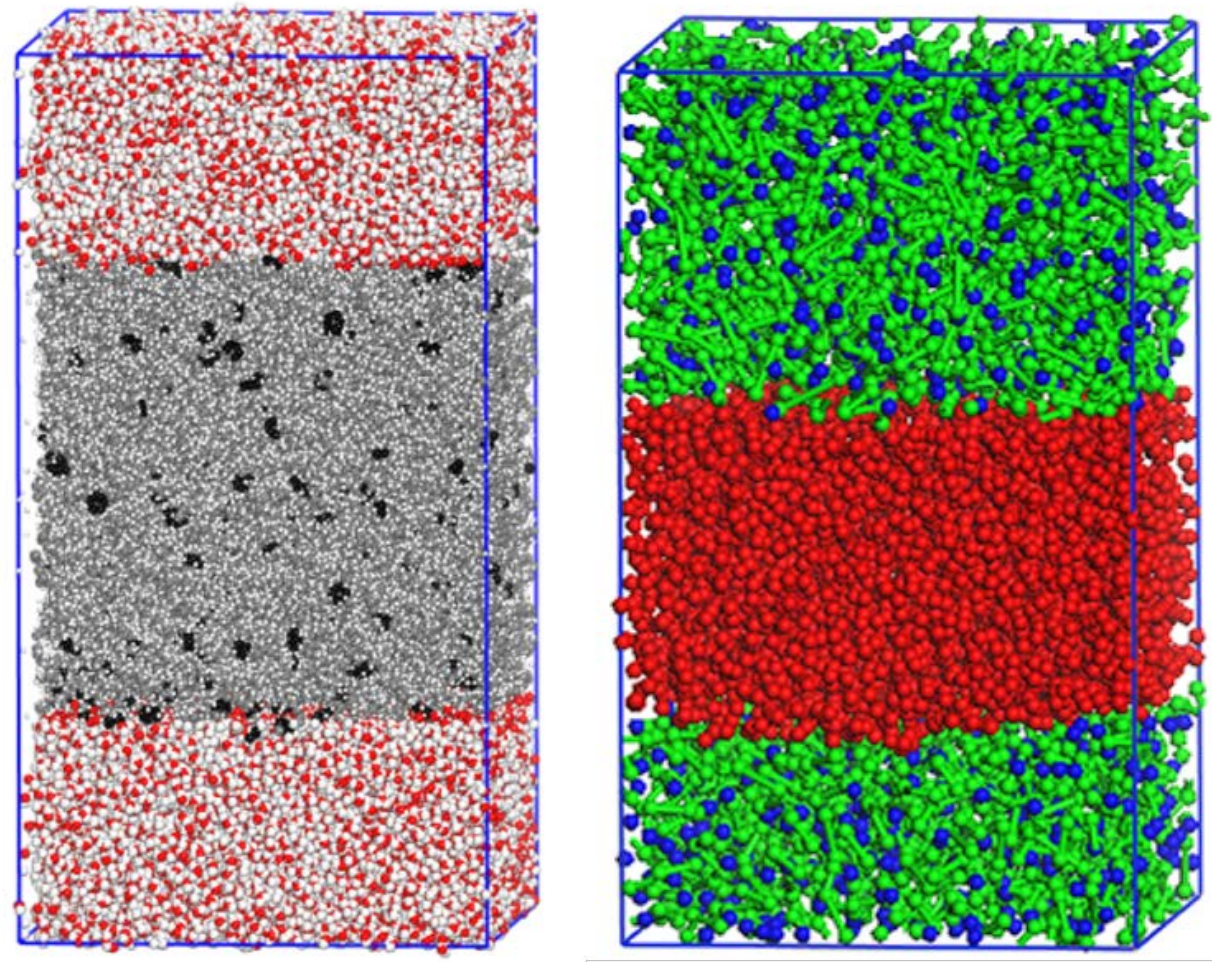

Fig. 1 Model of dodecane-toluene/water MD, left, and DPD, right. Color code: (left) O, red; H, white; C-dodecane, grey; C-toluene, black; (right) water, red; toluene, blue; dodecane, green.

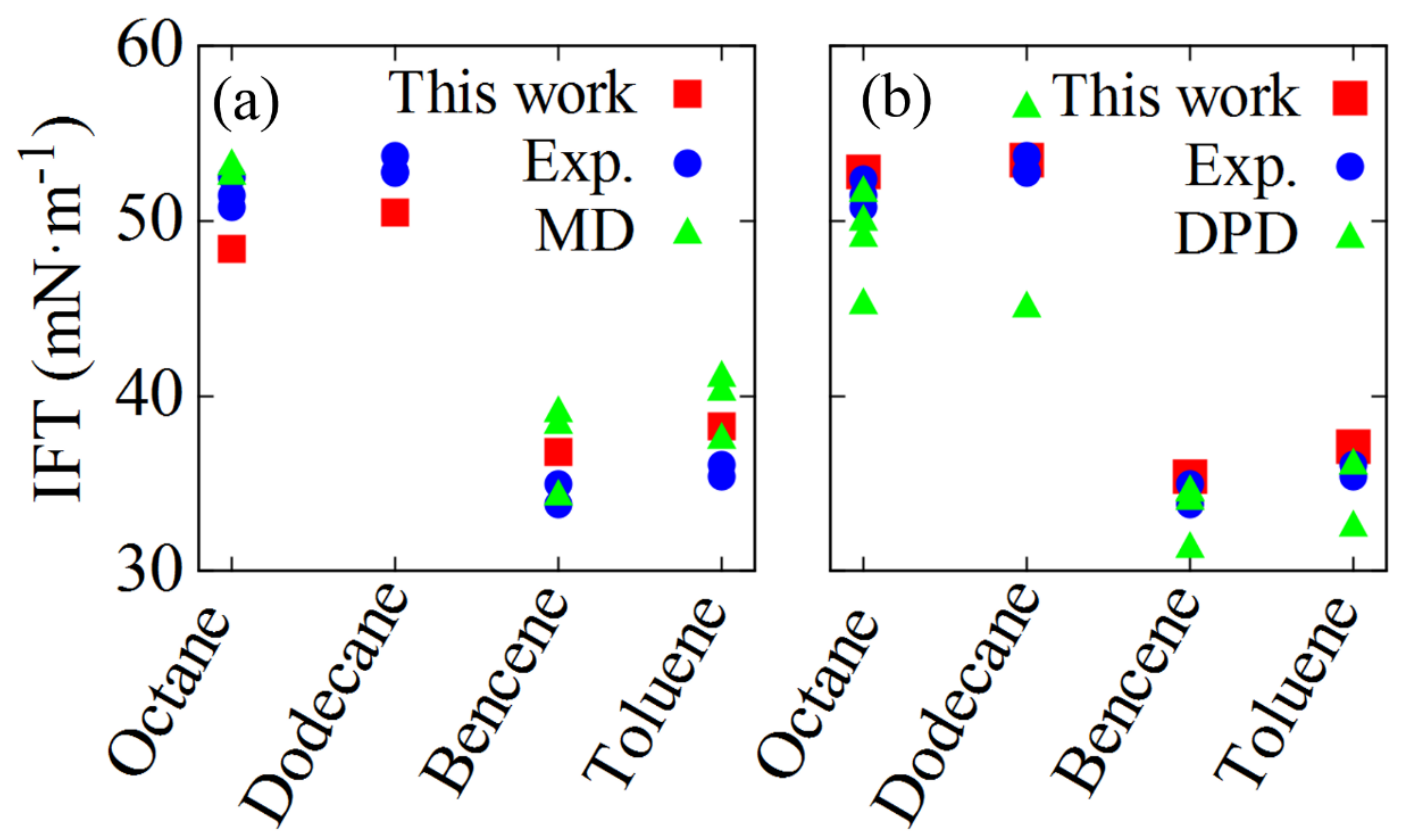

Fig. 2 IFT hydrocarbons/water using a) MD and b) DPD. 


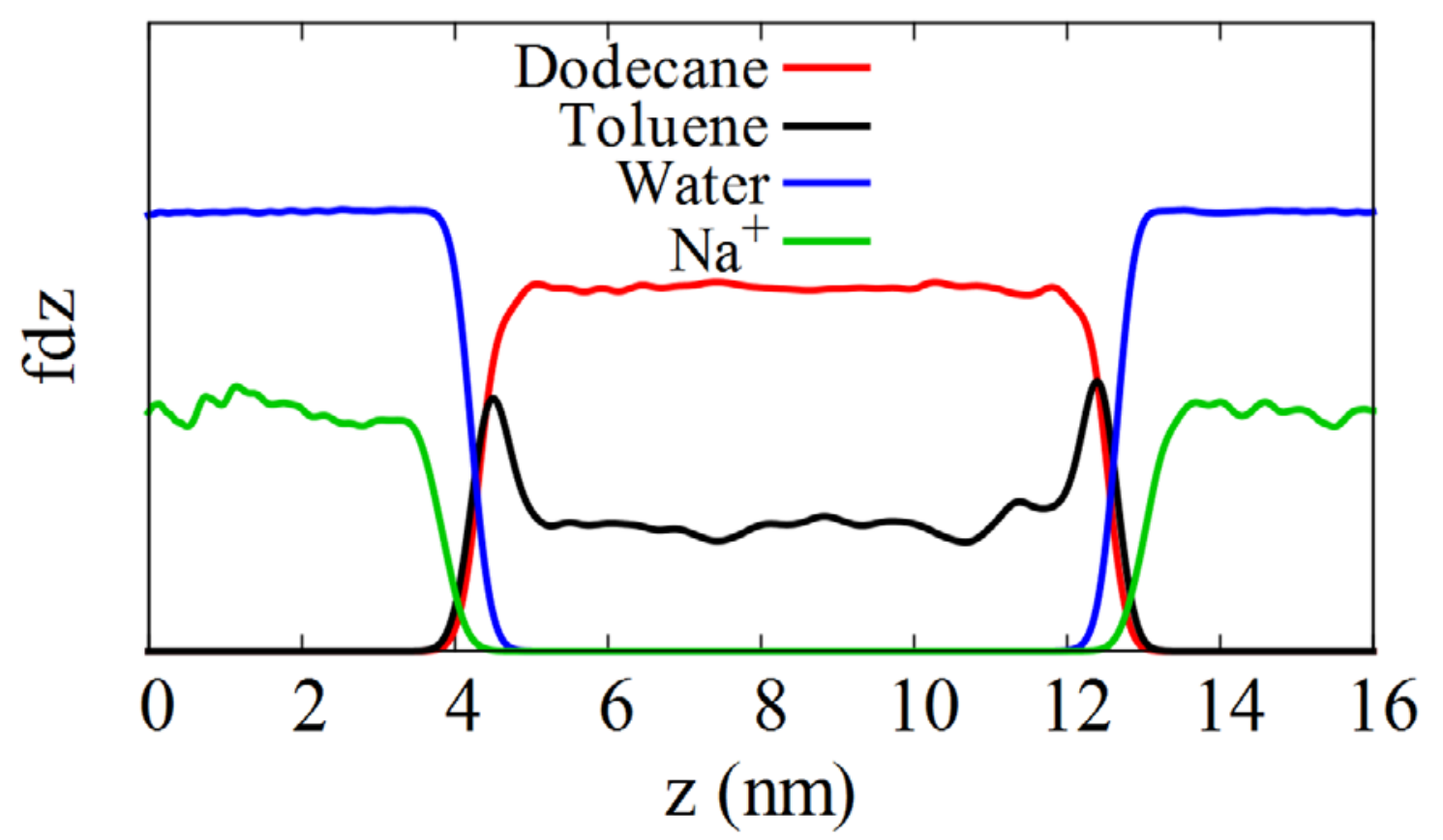

Fig. 3 Distribution function along $\mathrm{z}$ axis of dodecane-toluene/water model.
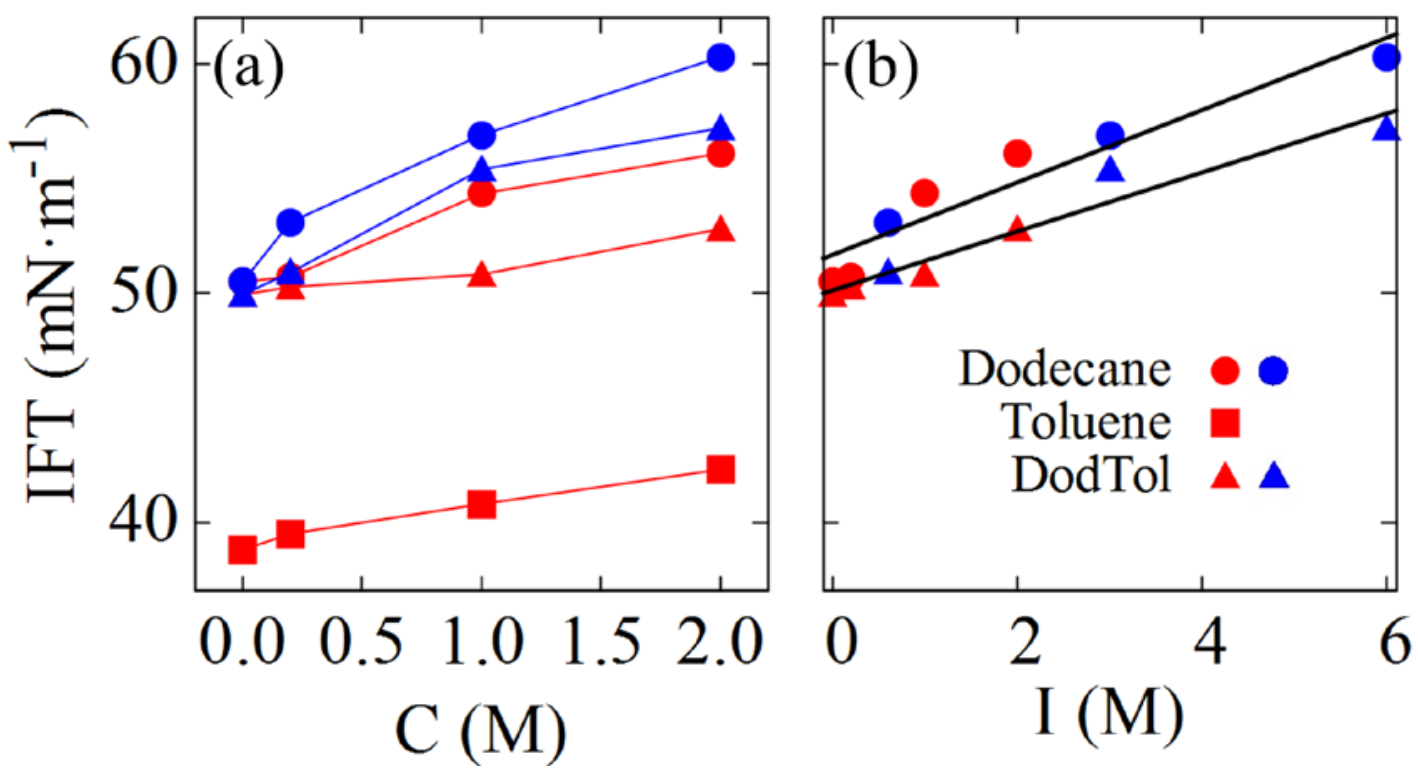

Fig. 4 a) IFT values of hydrocarbon/water vs brine concentration: $\mathrm{NaCl}$ (red) and $\mathrm{CaCl}_{2}$ (blue) b) IFT values vs ionic strength: $\mathrm{NaCl}$ (red) and $\mathrm{CaCl}_{2}$ (blue). 


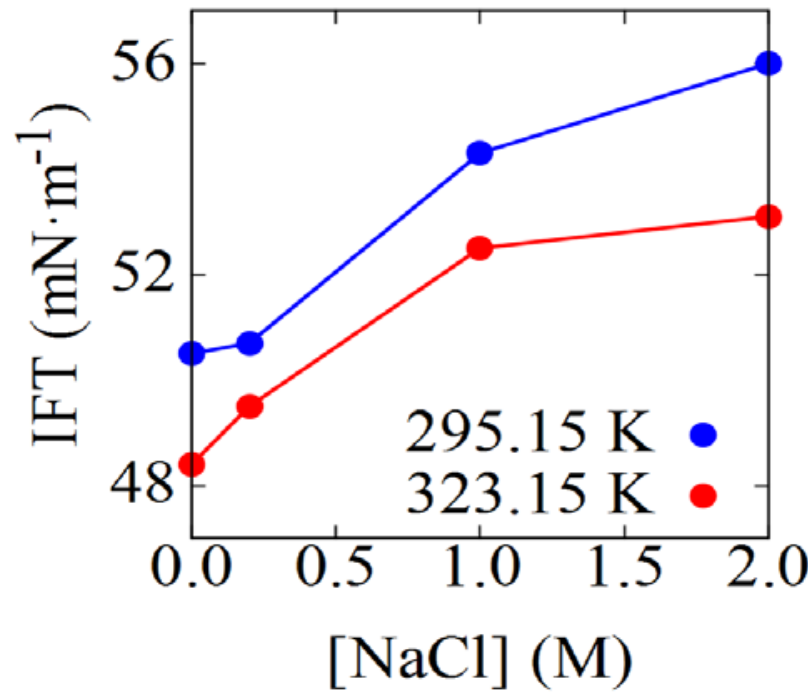

Fig. 5 IFT values for dodecane/water vs brine concentration $\mathrm{NaCl}$ at different temperatures. 


\section{References}

${ }^{1}$ Creton B, Nieto-Draghi C, Pannacci N (2012) OIL GAS J 67:969-982

${ }^{2}$ Marx D and Hutter J (2000) In Grotendorst J (ed) Modern methods and algorithms on quantum chemistry Vol 1\&3 John von Neumann Institute, NIC series

${ }^{3}$ Groot R D, Warren P B (1997) J Chem Phys 107:4423-4435

${ }^{4}$ Ikeda N, Aratono M, Motomura K (1992) J Colloid Interface Sci 149:208-215

${ }^{5}$ Cai BY, Yang JT, Guo TM (1996) J Chem Eng Data 41:493-496

${ }^{6}$ Aveyard R, Saleem SM (1976) J Chem Soc Faraday Trans 1, 72:1609-1617

${ }^{7}$ Vijapurapu CS, Rao DN (2004) Colloids Surf A 241:335-342

${ }^{8}$ Moeini F, Hemmati-Sarapardeh A, Ghazanfari M-H, Masihi M, Ayatollahi S (2014) Fluid Phase Equilibr 375:191-200

${ }^{9}$ Bai JM, Fan WY, Nan GZ, Li SP, Yu BS (2010) J Dispersion Sci Technol 31:551-556

${ }^{10}$ Isaacs E, Smolek K (1983) Can J Chem Eng 61:233-240

${ }^{11}$ Cheung DL (2012) Langmuir 28:8730-8736

${ }^{12}$ Stukan MR, Ligneul P, Boek ES (2012) Oil Gas Sci Technol 67:737-742

${ }^{13}$ Var Buuren AR, Marrink SJ, Berendsen HJC (1993) J Phys Chem 97:9206-9212

${ }^{14}$ Mikami Y, Liang Y, Matsuoka T, Boek ES (2013) Energy Fuels 27:1838-1845

${ }^{15}$ Kunieda M, Nakaoka, K, Liang Y, Miranda CR, Ueda A, Takahashi S, Okabe H, Matsuoka T (2010) J Am Chem Soc 132:18281-18286

${ }^{16}$ de Lara LS, Michelon MF, Miranda CR (2012) J Phys Chem B 116:14667-14676

${ }^{17}$ Sedghi M, Piri, M, Goual, L (2016) Langmuir 32:3375-3384

${ }^{18}$ Kirkwood J G, Buff F P (1949) J Chem Phys 17:338-343

${ }^{19}$ Plimpton S (1995) J Comp Phys 117:1-19

${ }^{20}$ Dauber-Osguthorpe P, Roberts V A, Osguthorpe D J, Wolff J, Genest M, Hagler A T (1988) Proteins 4:31-47

${ }^{21}$ Hockney and Eastwood (1989) Computer Simulation Using Particles, Adam Hilger, NY

${ }^{22}$ Nosé S, (1984) J Chem Phys 81:511-519

${ }^{23}$ Martínez L, Andrade R G, Birgin E, Martínez J M, (2009) J Comp Chem 30:2157-2164

${ }^{24}$ Dassault Systèmes BIOVIA, Materials Studio, 8.0, San Diego: Dassault Systèmes, 2014

${ }^{25}$ Hoogerbrugge PJ, Koelman JMVA (1992) Europhys Lett 19:155-160

${ }^{26}$ Groot RD, Warren PB (1997) J Chem Phys 107:4423-4435

${ }^{27}$ Maiti A, McGrother S, (2004) J Chem Phys 120:1594-1601

${ }^{28}$ Rezaei H, Modarress H (2015) Chem Phys Lett 620:114-112

${ }^{29}$ Goel H, Chandram P R, Mitra K, Majumdar S, Ray P (2014) Chem Phys Lett 600:62-67

${ }^{30}$ Andersson MP, Bennetzen M V, Klamt, A, Stipp S L (2014) J Chem Theory Comput. 10:3401-3408

${ }^{31}$ Zeppieri S, Rodríguez J, López de Ramos A L (2001) J Chem Eng Data 46:1086-1088

${ }^{32}$ Goebel A, Lunkenheimer K (1997) Langmuir 13:369-372

${ }^{33}$ Freitas AA, Quina F H, Carroll F A (1997) J Phys Chem B 101:7488-7493

${ }^{34}$ Backes HM, Ma JJ, Bender E, Maurer G (1990) Chem Eng Sci 45:275-286

${ }^{35}$ Centro de Tecnología Repsol S. A., to be published. 